\title{
Sexual Knowledge in Post-Myocardial Infarction Patients: A Cross-Sectional Study
}

Rida Farhan ${ }^{1}$, Rabail Yousuf ${ }^{2}$, Syeda Namayah Fatima Hussain ${ }^{3}$, Maaz Khan ${ }^{4}$, Zara Bilal ${ }^{5}$, Maryam Khan ${ }^{6}$, Zulekha Yousuf ${ }^{7}$, Burhan Khatri ${ }^{5}$, Ayesha Siddiqua ${ }^{8}$, Shenel A. Khan ${ }^{1}$

1. Medicine, Dow Medical College, Dow University of Health Sciences, Karachi, PAK 2. Cardiology, Dow Medical College, Dr. Ruth K. M. Pfau Civil Hospital, Karachi, PAK 3. Medicine, Liaquat National Hospital and Medical College, Karachi, PAK 4. Cardiology, United Medical and Dental College, Karachi, PAK 5. Medicine, Bahria University Medical and Dental College, Karachi, PAK 6. Internal Medicine, Ziauddin University, Karachi, PAK 7. Medicine, Jinnah Sindh Medical University, Karachi, PAK 8. Internal Medicine, Dow Medical College, Dow University of Health Sciences, Karachi, PAK

Corresponding author: Rabail Yousuf, rabail.yousuf@yahoo.com

\section{Abstract}

\section{Background}

Adequate sexual knowledge is a key component of cardiac rehabilitation. Sexual knowledge in postmyocardial infarction (MI) patients is unknown. Thus, we conducted this study to investigate the sexual knowledge of post-myocardial infarction patients and their accessibility to sexual counseling.

\section{Methodology}

Between July and September 2018, a cross-sectional survey was carried out in 6six major hospitals in Karachi. The non-probability convenient sampling technique was used to include all patients meeting the inclusion criteria. To reduce biases, face-to-face interviews were conducted by investigators who were trained prior to the start of data collection. Knowledge was assessed using the Swedish version of the "Sex after MI Knowledge Test" questionnaire, where a higher score meant higher knowledge. Data analysis was done using Statistical Package for Social Sciences (SPSS) version 21.0 (IBM Corp., Armonk, NY) The t-test was applied to compare the knowledge score between males and females.

\section{Results}

There was a total of 278 MI patients with a mean age of 54 years, of which $60 \%$ were men. The "Sex After MI Knowledge Test" scores varied between 37 and 67 (mean score $51 \pm 5$ ). None of the participants obtained the maximum possible test score of 75 . The most frequently occurring score was 49 (16\%). Around half of the participants (48\%) incorrectly believed that sex cannot be safely resumed within a few weeks after the heart attack. Limited knowledge was found in questions pertaining to alcohol, viagra, and late evening being the best time to have sex. Medical knowledge was provided to only $27 \%$ ( $n=76$ ) of the participants, of whom $77 \%$ $(n=58)$ received it from the hospital staff itself. A significant difference existed in scores obtained by males and females, with males having a higher score and the mean difference in scores being $1.7(\mathrm{p}=0.015)$.

Received 04/11/2020

Review began 05/29/2020 Review ended 05/31/2020 Published 06/06/2020

\section{() Copyright 2020}

Farhan et al. This is an open access article distributed under the terms of the Creative Commons Attribution License CC-BY 4.0., which permits unrestricted use, distribution, and reproduction in any medium, provided the original author and source are credited.

\section{Conclusion}

Lack of sexual knowledge in MI patients due to the inadequacy of healthcare providers and the social stigma surrounding the topic causes marital strain leading to low quality of life.

Categories: Cardiology, Public Health, Epidemiology/Public Health

Keywords: sexual knowledge, myocardial infarction

\section{Introduction}

It has been predicted that coronary heart disease (CHD) will be the cause of 11 million deaths by 2020 [1-2]. Considering these rising numbers, adequate treatment and recovery following a myocardial infarction (MI) have become increasingly important. It is crucial to maintain an acceptable quality of life and well-being following an MI, amongst which resuming one's sexual life is also a key. Fear of recurrence, exercise intolerance, and drugs causing sexual dysfunction have known to hinder a patient's sexual life following an MI [3]. Moreover, adequate sexual knowledge is also a key component of cardiac rehabilitation, as episodic physical and sexual activity is believed to be associated with an increased risk of MI [4].

Studies have shown that normal sexual activity causes a peak heart rate of 125 accompanied by a peak systolic blood pressure of 150-160 mmHg [5]. In MI patients, this increased heart rate adds to the workload of the heart and makes them more prone to a second attack. The drugs usually prescribed post-MI have side effects that impair sexual function, particularly in relation to erectile dysfunction in men [5-8]. Given the 
effect of these numerous post-MI conditions on the quality of life of patients, there is a need for them to understand the amount of physical activity, including sexual activity, could increase the chances of MI recurrence [9]. However, after hospital discharge, it is generally recommended to resume sexual activity unless stress testing reveals any signs of ischemia or arrhythmias.

Although many patients believe that sexual activity may lead to acute cardiac events, many hesitate to discuss their concerns with their physicians due to the sensitivity of the topic [10-11]. In addition, health care professionals are either uncomfortable about providing any sexual knowledge or they lack the relevant information and competence to communicate about the possible sex-related issues that their patient might face following an MI [12]. Therefore, there is also a need for clinicians to impart appropriate knowledge on the subject, to their patients following their hospital stay after an MI. A study suggested that only one-third of patients received any form of counseling after suffering from a cardiac event [13]. Since there is a paucity of data connecting the impact of MI to the sexual life of the patient, most studies in the past have been noted to focus on pharmacological interventions only, rather than also focusing on the sexual well-being of the individual [14]. Considering the lack of data on the subject, especially in Asia, the primary objective of this study was to investigate the sexual knowledge in patients who had suffered an MI and their accessibility to sexual counseling.

\section{Materials And Methods Design and setting}

This study was conducted over a period of 12 weeks from July to September 2018, to assess the sexual knowledge, awareness, and self-care practices among patients who had an MI in Karachi, Pakistan. This study was approved by the institutional review board of the Dow University of Health Sciences. A nonprobability convenience sample was recruited from the wards and outpatient departments of six tertiary care hospitals in Karachi, Pakistan. The inclusion criteria comprised adult patients who were diagnosed with MI a minimum of one month ago by a physician at a hospital and were married and in a sexual relationship with their spouse. The exclusion criteria comprised patients above 75 years of age, those who had MI within the last month, and patients suffering from any mental disability or known sexual problems.

\section{Data collection}

In this study, the Swedish version of the "Sex After MI Knowledge Test" was used, which was available in the English language and was also translated to Urdu for people who did not understand English [15]. This 25item instrument has moderate internal consistency and a Cronbach's alpha of 0.61 [15]. It is a self-rating questionnaire that evaluates the patient's knowledge regarding the resumption of sexual activity after suffering from an MI. A total of 35 questions were present in the questionnaire and consisted of questions regarding their sexual history and whether the participants had received any prior knowledge concerning sexual health and activity after MI. The second part of the questionnaire assessed the patient's knowledge concerning sexual activity after MI. It consisted of 25 questions, which were divided into three categories: category one was related to symptoms and physiological reactions during and after sexual activity, the importance of rest, the effect of sexual foreplay, masturbation, and oral and anal sex on heart, the correct time to resume sexual activity and its frequency; category two involved questions related to common emotional responses; and category three involved environmental influences such as medications, alcohol, meals, surrounding conditions, and timing and their effect on sexual activity after MI. In this section, the participants were given three choices (true, false, and do not know) for the statements given, upon which they were scored accordingly. Every correct response (have knowledge) received a score of three and every incorrect response as well as a response of 'do not know' was given a score of one. Thus, 75 was the maximum possible score generated with the scores ranging between 25 and 75 .

\section{Procedures and ethics}

The participants were explained the purpose of the study and verbal consent was obtained before proceeding with the questionnaire. To reduce biases, face-to-face interviews were conducted by the investigators, who were trained prior to the start of data collection. Standard medical terms were also defined and explained to the participants as to eliminate ambiguity. Questions were asked privately, away from other patients, so that they may be able to answer comfortably. They were assured that confidentiality would be maintained, considering the sensitivity of the topic in terms of cultural and social contexts. All interviewers used a standard protocol and any incomplete questionnaires were discarded. The questions were read out to the patients and their responses recorded. A total of 350 responses were collected, in excess of the calculated sample size of 278 . The calculated sample size was based on a $50 \%$ anticipated frequency from 'The 25 -item Sex after MI knowledge test' study at 95\% CI [15].

\section{Statistical methods}

The data were entered and analyzed using the Statistical Package for Social Sciences (SPSS) version 21.0 (IBM Corp., Armonk, NY). Frequencies and percentages were enumerated for categorical responses. The level of sexual knowledge was computed as a continuous variable. Differences in knowledge between males and females were measured as differences between the total scores using the t-test. In all cases, a p-value $<0.05$ 


\section{Cureus}

was considered significant.

\section{Results}

The demographic characteristics of the participants are shown in Table 1. A total of 278 participants were included in this study, of which $60 \%$ were men. The mean age was found to be $54 \pm 8$ years. Most of the participants had received school-level education (56\%), with only $17 \%$ having studied past university level. Ninety-three percent of the participants felt it was "always" or "sometimes" important to have sex. Many of them reported the frequency of having sex as monthly (48\%) while $29 \%$ experienced discomfort during intercourse. Medical knowledge was provided to only $27 \%(n=76)$ of the participants, of whom $77 \%(n=58)$ received it from the hospital staff itself. 


\section{Cureus}

Background Characteristics

Age, mean $\pm S D$, years

Sex

Male

Female

Education

No education

Up to school

University

Years of relationship with partner

5 to 20 years

20 to 40 years

40 to 50 years

Above 50 years

Importance of having sex

Always important

Sometimes important

Never important

Frequency of sex in last 2 months

Monthly

Weekly

Never

Discomfort during intercourse

Yes

No

Presence of symptoms

Always

Sometimes

Never

Medical knowledge regarding sexual activity after MI

Yes

By hospital faculty

Family and friends

Self-awareness

No
$27(10)$

$112(40)$

Participants $\mathrm{n} \pm$ SD or (\%)

$54 \pm 8$

$166(60)$

$112(40)$

76 (27)

155 (56)

47 (17)

43 (15)

202 (73)

27 (10)

$6(2)$

134 (48)

125 (45)

19 (7)

132 (48)

76 (27)

70 (25)

80 (29)

$198(71)$

$139(50)$

76 (27)

58 (77)

9 (12)

9 (11)

202 (73)

\section{TABLE 1: Demographic characteristics}

SD: standard deviation; MI: myocardial infarction 


\section{Cureus}

The "Sex After MI Knowledge Test" scores ranged from 37 - 67 (mean score $51 \pm 5$ ). No participant managed to obtain the maximum possible test score of 75 in the test, with the most frequently occurring score being 49 (16\%). Table 2 shows the results of questions related to symptoms and physiological reactions during intercourse. Emotional reactions and other influencing factors (medications and environmental factors) are shown in Table 3 and Table 4, respectively. The highest scores were obtained on the question "If you have chest pain during sex, you should stop and rest," $89 \%$ of the people correctly answered "True." The statements "Masturbation and oral sex are more harmful to the heart than sexual intercourse" and "Anal intercourse can be resumed just as vaginal intercourse since it has less effects on the heart" were answered correctly by only $8 \%$ and $7 \%$ of the participants, respectively.

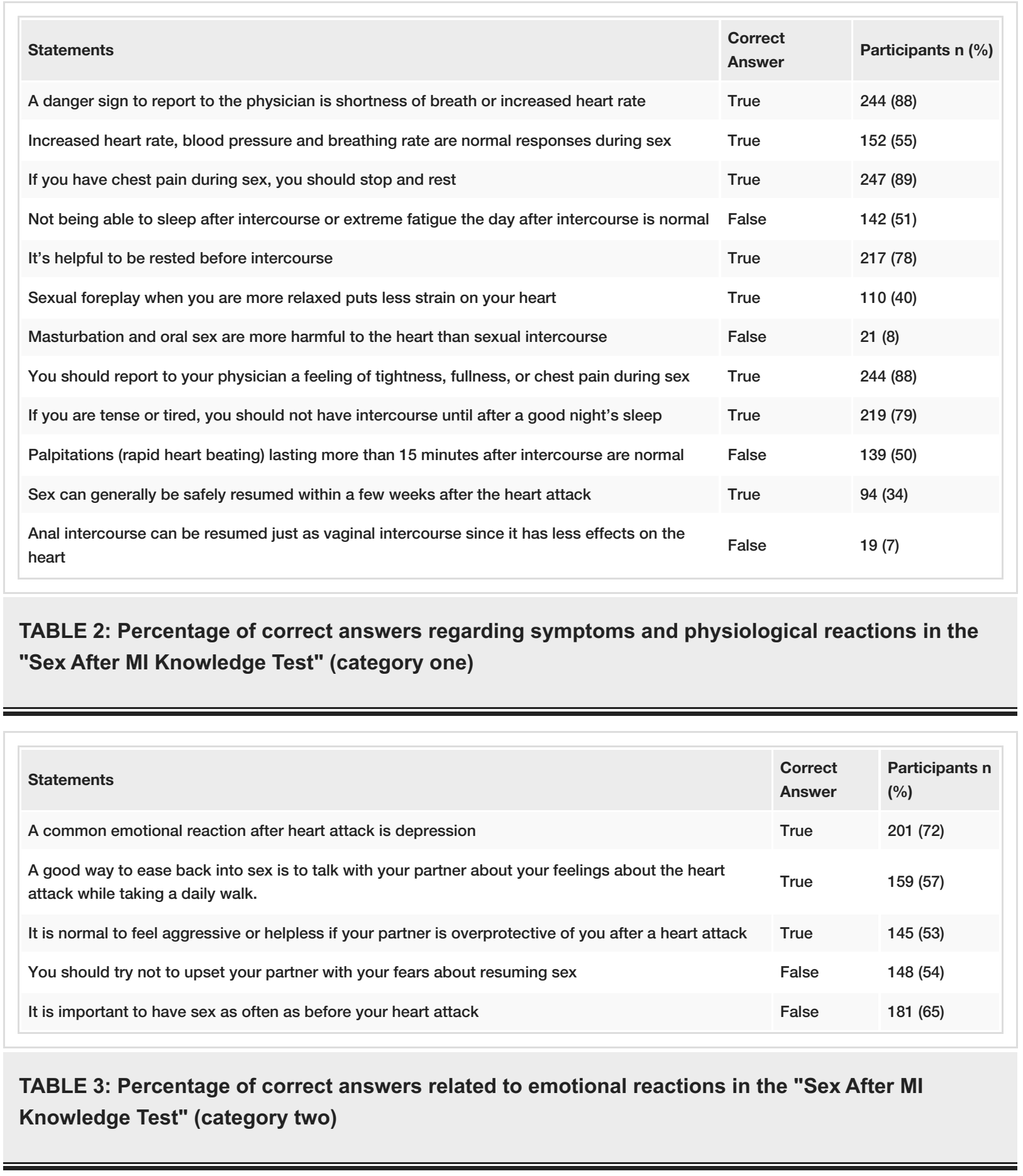




\section{Cureus}

Statements

Drinking alcohol prior to sex will help you relax and improve sex.

Some medicines used for high blood pressure, anxiety, or depression can affect sex.

If you think a medicine is causing a problem with sex, you should stop it immediately

Late evening or the end of the day is the best time to have sex when you are more relaxed.

Wait 2-3 hours after a heavy meal before having sex

A room temperature that is not too hot or cold is important for sex

Such precautionary measures are helpful

Do you think taking drugs like Viagra for erection are helpful
Participants n (\%)

False

True

$134(48)$

False

$117(42)$

False

$27(10)$

True

220 (79)

True

185 (67)

True

208 (75)

True

37 (13)

TABLE 4: Percentage of correct answers related to environmental and other influencing factors in the "Sex After MI Knowledge Test" (category three)

Almost half (48\%) of the participants incorrectly believed that it was unsafe to resume sex after a few weeks following an MI. Moreover, 18\% of them did not know whether this statement was correct or not. Concerning environmental factors, the least amount of knowledge was shown in questions pertaining to alcohol (13\%), phosphodiesterase 5 inhibitors such as sildenafil (Viagra) (13\%), and late evening being the best time to have sex (10\%). Overall, the participants were most knowledgeable in category two (emotional reactions) where $61 \%$ of the answers obtained were correct. In category one (symptoms and physiological reactions), 55\% of the questions were answered correctly. Less than half of the answers in category three (environmental and other influencing factors) were answered correctly (43\%), showing the least amount of knowledge about environmental and other factors such as medications and alcohol use affecting sex (Figure 1). Figure 2 shows the percentage of correct responses by both genders. A significant difference was found in scores obtained by males and females (mean difference in scores was $1.7 ; \mathrm{p}=0.015$ ).

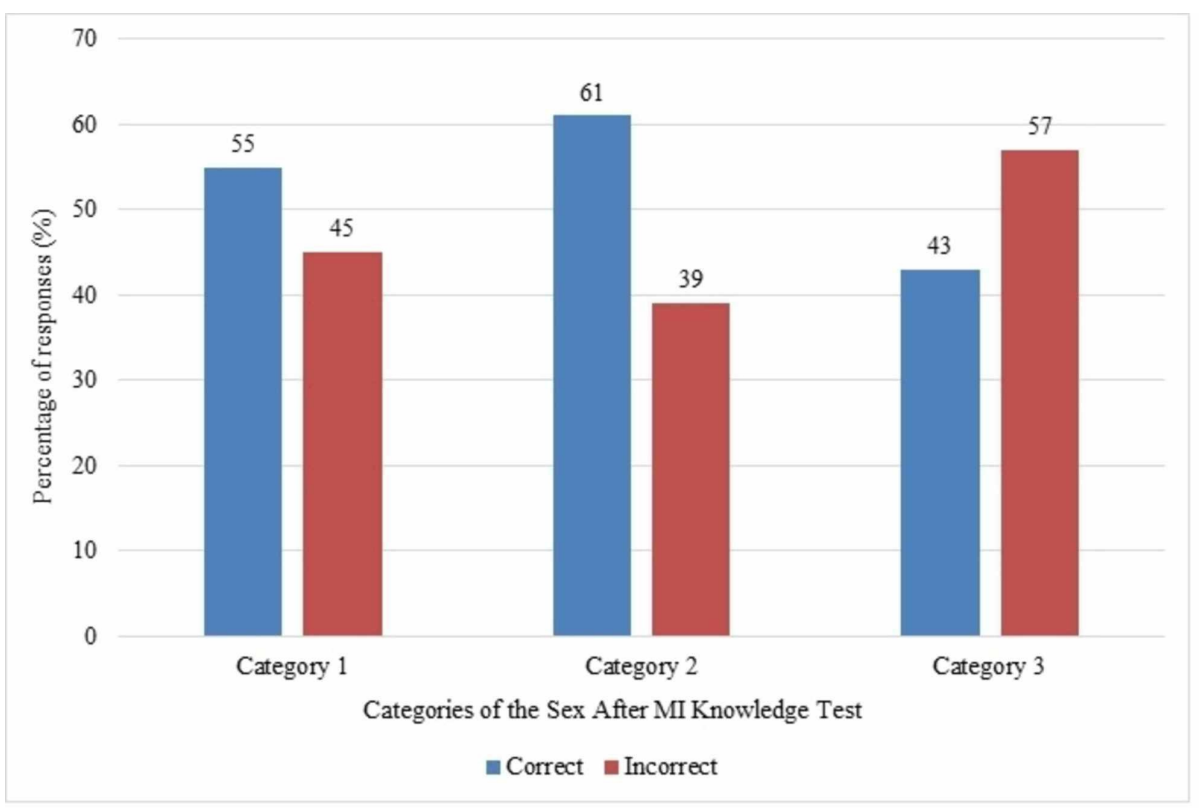

FIGURE 1: Percentage of correct and incorrect answers in each category of the "Sex After MI Knowledge Test" 


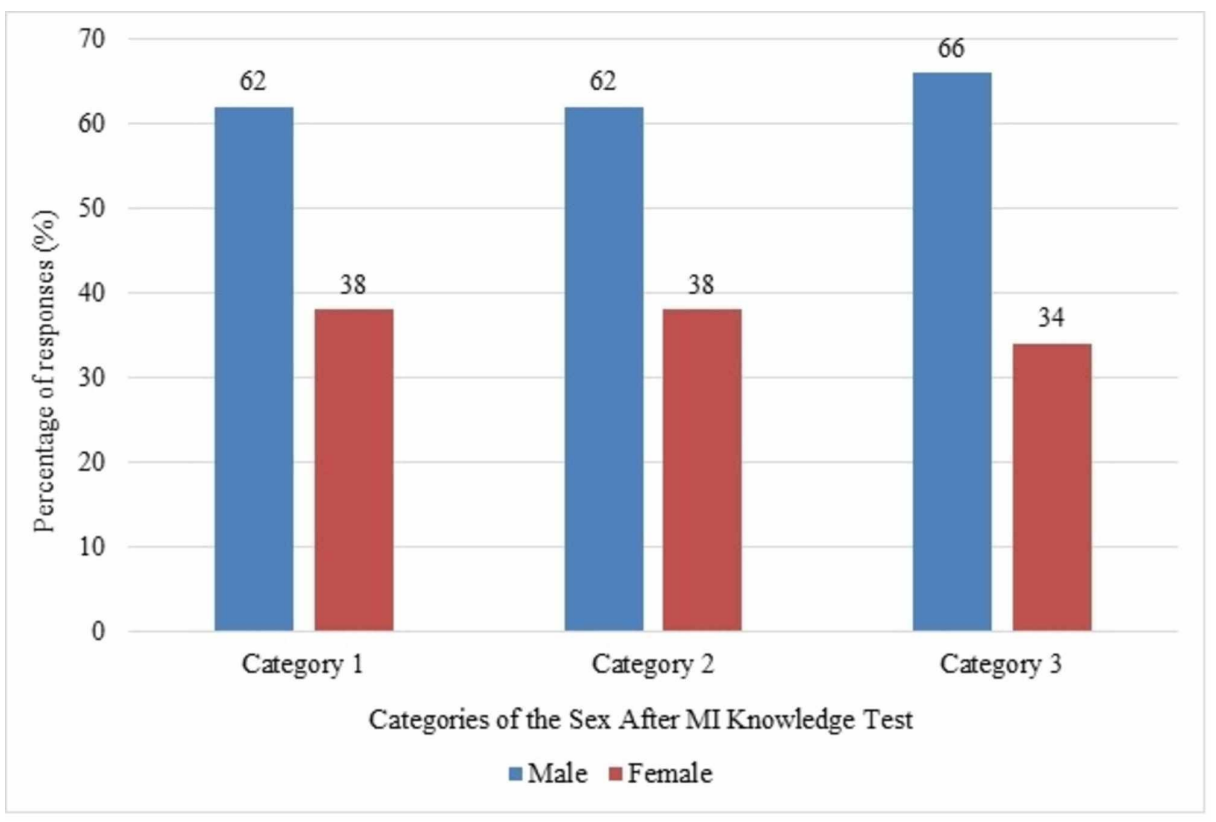

FIGURE 2: Percentage of correct responses of both genders

\section{Discussion}

We found that most of the participants had limited knowledge about resuming sexual activity post-MI. Any form of sexual counseling by healthcare professionals was lacking. A previous study conducted showed similar results with a mean score of $51 \pm 10$, and another study, the Swedish national survey, found that healthcare professionals rarely provided any information regarding sexual activity to post-MI patients or their partners [16-17]. Sexual counseling or openly discussing sexual activity is often considered a shameful topic and is also an old societal taboo, especially in Asian countries like Pakistan. In our study, most participants were noted to be either uneducated or educated up to only the school level, reflective of low socioeconomic status. Therefore, due to the unavailability of resources like the internet or healthcare books, the medical team remains the sole source of guidance regarding any health-related queries they might have.

Furthermore, the mean age of the participants with a history of MI was 54 years; an age where the sexual life of most becomes stagnant. Most people of this age, especially those from the lower socioeconomic background, have either already achieved the status of grandparents or are about to become grandparents. Moreover, across the Indian subcontinent, particularly in Pakistan, the joint family system, which consists of many generations living under the same roof, still prevails, which makes undertaking sexual activity for these relatively older individuals an embarrassing task to engage in. Hence, for the reasons mentioned above, most individuals do not feel the need to be sexually counseled or ask for information following an MI.

We found men to have significantly greater knowledge on the subject than women. Several studies have concluded that women were significantly less informed and less likely to receive sexual counseling and that men had comparatively higher knowledge than women regarding the subject [17-19]. This is because, in a patriarchal society, sex is an easily spoken subject among men. It is considered to be a conquest and a matter of pride for them, which is supported by several studies stating that men were generally more curious than women regarding obtaining information about sexual concerns [20-21]. Another reason is that more men suffer from MI (at up to 60 years) as compared to women [22]. Another study showed that since women with coronary artery diseases belonged to a much older age group as compared to men, they were less likely to discuss the matter related to sexual activity with their doctors, who also assume them to be sexually inactive, thus ignoring the need for sexual counseling [23-25]. These findings demonstrate the need for clinicians to provide sufficient information to both the genders equally about resuming sexual activity after MI.

Due to the common knowledge of the physical signs and symptoms of MI, the majority of the participants were aware of chest pain during sex as a danger sign and understood the need to stop and rest. However, owing to the considerable secrecy and mortification associated with sexual intercourse, along with the reluctance of healthcare staff to broach the subject with their patients, almost half of the patients were hesitant to resume sex within the few weeks following an MI. A previous study conducted in Brazil also found that only $4 \%$ of the patients received sexual guidance and more than half were doubtful of the acceptability of sexual activity after being discharged from the hospital [26]. This was mostly due to the dread of having re-infarction or even sudden death following any sexual activity [27]. As a consequence, patients are known to suffer from stress, anxiety, and depression, putting a strain on their marital life. 
Sexual counseling after an MI is an important factor for recovery, as it helps the patients to ease into their daily routines, avoiding feeling crippled due to an inability to perform well in their sex life [28]. Some other findings from the data collected were limited knowledge pertaining to oral or anal sex and drinking alcohol. This could be because of the majority's religious beliefs against these topics, prompting an unwillingness to answer such questions. There was also a lack of knowledge about medications and their effects on the heart. The results of this study also disclosed that the majority of the participants chose to readily give up on medication over temporary problems in their sexual activity, hence reflecting upon the personal importance of a healthy sexual life.

Medical knowledge was provided to only $27 \%(n=76)$ of the participants, of whom $77 \%(n=58)$ received it from the hospital staff. Keeping in view the lack of other sources of information available to these patients, it is essential to train healthcare staff to be better equipped to counsel patients and to encourage them to discuss their doubts more openly. The "Sex After MI Knowledge Test" itself can be an effective tool in not only initiating this discussion but also in giving useful insights as to where the patient lacks awareness. They should be sufficiently reassured that sexual activity is neither a risk factor nor a trigger in inducing another MI, thus alleviating their concerns and facilitating complete recovery [10]. Another way of creating awareness could be the use of informative leaflets and guidelines distributed by clinicians alongside group discussions led by informed healthcare staff [16]. Telephone counseling is also emerging as an effective means of addressing the sexual concerns of the patients while maintaining their anonymity; this, alongside the use of leaflets, could prove to be particularly useful in conservative countries [22].

There are several limitations in our study that should be taken into consideration. First, the data collected were mostly from people of low socioeconomic backgrounds and from government hospitals. Although these are large tertiary care hospitals with people from all over the country being treated there, we believe that including private sector cardiac care institutes would have been a better representative sample. Second, since face-to-face interviews were conducted and a match in sex between the interviewer and participant was not considered, there is a possibility that information was witheld considering the sensitive nature of the topic. Third, since alcohol and anal intercourse are prohibited in the state religion, the results are likely to not be an accurate representation. Fourth, we did not assess the correlation between sexual knowledge and the time duration after MI, nor did we study the sexual knowledge of the partners of MI patients. In the future, detailed studies that include more hospitals and those including the knowledge of both the patient and their partners should be conducted.

\section{Conclusions}

This study was conducted to gain insight into the sexual knowledge of patients who had suffered a myocardial infarction (MI). Owing to the shame associated with anything related to sexual intercourse, MI patients are often deprived of proper counseling after an attack. Given that an inactive sexual life can cause marital problems, disrupting the entire family structure, it is essential for healthcare providers to guide MI patients in terms of sexual activity following their hospital stay. We believe that removing the patient's fear and doubts about resuming sexual intercourse would help hasten their recovery and alleviate any undue stress caused between them and their partners, hence improving their quality of life.

\section{Additional Information \\ Disclosures}

Human subjects: Consent was obtained by all participants in this study. Animal subjects: All authors have confirmed that this study did not involve animal subjects or tissue. Conflicts of interest: In compliance with the ICMJE uniform disclosure form, all authors declare the following: Payment/services info: All authors have declared that no financial support was received from any organization for the submitted work. Financial relationships: All authors have declared that they have no financial relationships at present or within the previous three years with any organizations that might have an interest in the submitted work. Other relationships: All authors have declared that there are no other relationships or activities that could appear to have influenced the submitted work.

\section{References}

1. World Health Organization. Cardiovascular diseases (CVDs) factsheet. (2017). Accessed: March 13, 2020: http://www.who.int/mediacentre/factsheets/fs317/en/.

2. Murray CJ, Lopez AD, World Health Organization: The Global Burden of Disease. A Comprehensive Assessment of Mortality and Disability From Diseases, Injuries, and Risk Factors in 1990 and Projected to 2020: Summary. The Harvard School of Public Health, Boston, MA; 1996.

3. Jaarsma T, Steinke EE, Gianotten WL: Sexual problems in cardiac patients: how to assess, when to refer . J Cardiovasc Nurs. 2010, 25:159-164. 10.1097/JCN.0b013e3181c60e7c

4. Dahabreh IJ, Paulus JK: Association of episodic physical and sexual activity with triggering of acute cardiac events: systematic review and meta-analysis. JAMA. 2011, 305:1225-1233. 10.1001/jama.2011.336

5. Falk RH: The cardiovascular response to sexual activity: do we know enough? . Clin Cardiol. 2001, 24:271275. 10.1002/clc.4960240403

6. Bacon CG, Mittleman MA, Kawachi I, Giovannucci E, Glasser DB, Rimm EB: Sexual function in men older than 50 years of age: results from the health professionals follow-up study. Ann Intern Med. 2003, 139:161- 
168. 10.7326/0003-4819-139-3-200308050-00005

7. Baumhäkel M, Schlimmer N, Kratz M, Hackett G, Jackson G, Böhm M: Cardiovascular risk, drugs and erectile function-a systematic analysis. Int J Clin Pract. 2011, 65:289-298. 10.1111/j.1742-1241.2010.02563.x

8. Araujo AB, Durante R, Feldman HA, Goldstein I, McKinlay JB: The relationship between depressive symptoms and male erectile dysfunction: cross-sectional results from the Massachusetts Male Aging Study. Psychosom Med. 1998, 60:458-465. 10.1097/00006842-199807000-00011

9. Steinke EE, Mosack V, Barnason S, Wright DW: Progress in sexual counseling by cardiac nurses, 1994 to 2009. Heart Lung. 2009, 40:e15-e24. 10.1016/j.hrtlng.2010.10.001

10. Levine GN, Steinke EE, Bakaeen FG, et al.: Sexual activity and cardiovascular disease: a scientific statement from the American Heart Association. Circulation. 2012, 125:1058-1072. 10.1161/CIR.0b013e3182447787

11. Lindau ST, Abramsohn EM, Bueno H, et al.: Sexual activity and counseling in the first month after acute myocardial infarction among younger adults in the United States and Spain. A prospective, observational study. Circulation. 2014, 130:2302-2309. 10.1161/CIRCULATIONAHA.114.012709

12. Doherty S, Byrne M, Murphy AW, McGee HM: Cardiac rehabilitation staff views about discussing sexual issues with coronary heart disease patients: a national survey in Ireland. Eur J Cardiovasc Nurs. 2011, 10:101-107. 10.1016/j.ejcnurse.2010.05.002

13. Lindau ST, Abramsohn E, Gosch K, et al.: Patterns and loss of sexual activity in the year following hospitalization for acute myocardial infarction (a United States national multisite observational study). Am J Cardiol. 2012, 109:1439-1444. 10.1016/j.amjcard.2012.01.355

14. Lange RA, Levine GN: Sexual activity and ischemic heart disease . Curr Cardiol Rep. 2014, 16:445. 10.1007/s11886-013-0445-4

15. Svedberg P, Johansson I, Persson S, et al.: Psychometric evaluation of 'The 25-item Sex after MI Knowledge Test' in a Swedish context. Scand J Caring Sci. 2012, 26:203-208. Accessed: March 13, 2020: https://onlinelibrary.wiley.com/doi/abs/10.1111/j.1471-6712.2011.00909.x. 10.1111/j.14716712.2011.00909.x

16. Brännström M, Kristofferzon ML, Ivarsson B, Nilsson UG, Svedberg P, Thylén I: Sexual knowledge in patients with a myocardial infarction and their partners. J Cardiovasc Nurs. 2014, 29:332-339. 10.1097/JCN.0b013e318291ede6

17. Ivarsson B, Fridlund B, Sjöberg T: Information from health care professionals about sexual function and coexistence after myocardial infarction: a Swedish national survey. Heart Lung. 2009, 38:330-335. 10.1016/j.hrtlng.2009.01.001

18. Rundblad L, Zwisler AD, Johansen PP, Holmberg T, Schneekloth N, Giraldi A: Perceived sexual difficulties and sexual counseling in men and women across heart diagnoses: a nationwide cross-sectional study. J Sex Med. 2017, 14:785-796. 10.1016/j.jsxm.2017.04.673

19. Bedell SE, Duperval M, Goldberg R: Cardiologists' discussions about sexuality with patients with chronic coronary artery disease. Am Heart J. 2002, 144:239-242. 10.1067/mhj.2002.124053

20. Bdair IAA, Abushaikha L: Jordanian patients knowledge regarding sexual health following coronary artery diseases. International Journal of Healthcare and Medical Sciences. 2018, 6:90-98.

21. Steenkamp JB, De Jong MG, Baumgartner H: Socially desirable response tendencies in survey research . J Mark Res. 2010, 47:199-214. 10.1509/jmkr.47.2.199

22. Simonelli C, Tripodi F, Cosmi V, Rossi R, Fabrizi A, Silvaggi C, Di Pomponio I: What do men and women ask a helpline on sexual concerns? Results of an Italian telephone counselling service. Int J Clin Pract. 2010, 64:360-370. 10.1111/j.1742-1241.2009.02269.x

23. Campbell DJ: Why do men and women differ in their risk of myocardial infarction? . Eur Heart J. 2008, 29:835-836. 10.1093/eurheartj/ehn074

24. Assari S: Intercourse avoidance among women with coronary artery disease . J Sex Med. 2014, 11:1709-1716. 10.1111/jsm. 12459

25. D'Eath M, Byrne M, Doherty S, McGee H, Murphy AW: The cardiac health and assessment of relationship management and sexuality study: a qualitative inquiry of patient, general practitioner, and cardiac rehabilitation staff views on sexual assessment and counseling for cardiac patients. J Cardiovasc Nurs. 2013, 28:E1-E13. 10.1097/JCN.0b013e318281d0b3

26. Lindau ST, Abramsohn E, Bueno H, et al.: Sexual activity and function in the year after an acute myocardial infarction among younger women and men in the United States and Spain. JAMA Cardiol. 2016, 1:754-764. 10.1001/jamacardio.2016.2362

27. Lunelli RP, Rabello ER, Stein R, Goldmeier S, Moraes MA: Sexual activity after myocardial infarction: taboo or lack of knowledge? [Article in Portuguese]. Arq Bras Cardiol. 2008, 90:172-176. 10.1590/s0066$782 \times 2008000300003$

28. Bispo GS, de Lima Lopes J, de Barros AL: Cardiovascular changes resulting from sexual activity and sexual dysfunction after myocardial infarction: integrative review. J Cardiovasc Nurs. 2013, 22:3522-3531. 10.1111/jocn. 12356 